\title{
An Analysis of the Spiritual Characteristics of the Females in the Novels of Writer Zhang Ailing
}

\author{
Ren Meihua, Xiao Jiugen* \\ Jiangxi Normal University, Research Center of Language and Language Life, Nanchang, China
}

Email address:

1437654809@qq.com (Ren Meihua),jxsdxjg666666@sina.com (Xiao Jiugen)

${ }^{*}$ Corresponding author

To cite this article:

Ren Meihua, Xiao Jiugen. An Analysis of the Spiritual Characteristics of the Females in the Novels of Writer Zhang Ailing. Science Innovation. Vol. 9, No. 1, 2021, pp. 7-11. doi: 10.11648/j.si.20210901.12

Received: February 1, 2021; Accepted: February 27, 2021; Published: March 4, 2021

\begin{abstract}
Most of Eileen Chang's novels describe her living environment, attitude towards life, marriage and hardship from the perspective of women. Their descriptions are thorough and meticulous, creating vivid female artistic images, which play a very important role in the history of modern Chinese literature. Writers' artistic shaping of women's images in the 1930s and 1940s in old China can be said to be a great contribution to the reappearance of Chinese women's spiritual characteristics. From all kinds of female artistic images created by writers' novels, such as money supremacy, silence and helplessness, and tragedy of marriage and love, people can clearly see through their specific and distinct female spiritual characteristics. These female images not only have far-reaching influence on Chinese literature, but also have great influence in the world humanities field. In order to thoroughly grasp the characters' images, we analyze the spiritual characteristics of all kinds of women in the works by comparative methods, and reflect on the writer's growth process, thus drawing a conclusion that the creation of female images in the works is closely related to the writer's family background and social environment at that time, which is essentially an artistic reflection of the social reality in that period.
\end{abstract}

Keywords: Zhang Ailing's Novels, Female image, Mental Characteristics

\section{作家张爱玲小说女性形象精神特性分析}

\section{任美花, 肖九根 ${ }^{*}$}

江西师范大学语言与语言生活中心, 南昌, 中国

\section{邮箱}

1437654809@qq.com（任美花），jxsdxjg666666@sina.com（肖九根）

\begin{abstract}
摘要: 张爱玲的小说, 大多都是从女性角度对其生存环境、人生态度、婚恋状况、生活困苦等方面进行描写的, 其刻 画深入细致, 创造了一个个栩栩如生的女性艺术形象, 在中国现代文学史上具有十分重要的地位。作家对旧中国二十 世纪三、四十年代女性形象的艺术塑造, 可以说是对中国女性精神特性再现的一大贡献。从作家小说塑造的诸如金钱 至上、沉默无助、婚恋悲剧等各式各样的女性艺术形象中, 人们可以清楚地透视其具体可感的非常鲜明的女性精神特 性, 这些女性形象不仅对中国文学有着十分深远的影响, 而且在世界人文领域里也产生了较大的影响力。为了透彻把 握人物形象, 我们以比较的方法分析作品中各类女性的精神特质, 并以此反观作家的成长历程, 从而得出了作品女性 形象的塑造与作家其时所处的家庭背景及社会环境均有十分密切的关系，实质上是那个时期社会现实的艺术反映。
\end{abstract}

关键词: 张爱玲小说, 女性形象, 精神特性 


\section{1. 引言}

张爱玲是一位才华超众而命运多外的女作家，她以无 与伦比的细淢情感, 勾画出一个迷人而难忘的世界。就像 作品《传奇》一样, 张爱玲也是一位传奇女性。她出生于 一个有名的家庭, 但她的生活却跌宕起伏。

她所勾画的迷人世界像是天上的雪月, 完美地展现出 社会中的各种人性。人性是文学的根底。作家所形容的人 性张力, 创造了一种悲剧性的意境。它不是夸耀的虚荣, 而是真正的恶浊, 其作品是对现实阴暗面的控告。本文旨 在探讨作家张爱玲小说所塑造的女性形象, 通过剖析其女 性形象, 力图揭示其笔下人物艰深人性的洞察力。作家创 造女性形象的基调是宏伟的, 有着艰深、哀痛、沉重的蕴 涵。其塑造人物形象的深刻性不亚于中国社会主流思想家 的深刻性。作家塑造的人物形象, 不仅对中国文学有着深 远的影响, 而且在世界人文领域内也产生了较大的影响。 作家小说中的人物形象艺术鬼力有着非常强的感染力, 一 个庞大而复杂的女性艺术形象群体, 经作家妙手架构就很 完美地責立起来了, 而且这一典型的形象群体就是二十世 纪三、四十年代社会女性形象的艺术再现。

关于作家作品中的女性形象，学者们以不同视角作过 多方面的分析。诸如间俊玲 (2015) 将其作品塑造的女性 形象与奥斯汀的作比较, 揭示其形象的共同点与不同点 [1]; 张冬梅（2016）着重剖析了作品中女性“神经质”的性 格以及作家性格对其创作所产生的影响 [2]; 而吴敬玲

（2017）则通过爱情的虚无与消解、亲情的缺失与沦丧、 友情的虚伪与背叛来揭示女性的悲剧命运及其人性异化 [3]。此外, 高天瑞（2019）还探视了作家作品中不同女性 的觉醒意识, 认为作家发出了与世界顶级女权主义者同样 震耳发聩的呐喊声[4]。

\section{2. 作家笔下女性形象的精神特质}

张爱玲个人婚姻的不幸对她后期女性意识的形成与 深化有着重要的影响。纵观张爱玲的一生, 她自始至终都 是一个经济独立的女性。无论是第一次与胡兰成的婚姻, 还是五十年代赴美的第二段婚姻, 她充当的都是经济独立 的角色, 甚至处在担负家计的主要地位。女性经济的独立 是其产生女性意识的基础。

在第一段婚姻中, 张爱玲扮演的是大多人以夫为天、 逆来顺受的传统女性角色。她抱持着中国传统的女性观, 对丈夫完全依赖和崇拜, “见了他, 她变得很低很低, 低 到尘土里。”[5]在跟胡兰成的婚姻中, 她除了经济独立之 外, 在情感上是一个依附的角色。虽然深爱胡兰成, 但是 她在盲目的爱情中迷失了自己, 也放弃了自己。即使面对 丈夫流亡出轨的现状, 她都能再三隐忍。因为思念丈夫, 她远赴温州山村探望, 在那里她忍受着委屈, 背负着被抛 弃的屈辱, 竟跟范秀美见面, 而且三人相安无事地住了一 段日子。“饱经创伤、痛苦异常的张爱玲对他十分绝望, 最 终与胡兰成决裂。”[6]在跟胡的分手诀别信里, 她还是低 眉顺目地写到: “我已经不喜欢你了, 你是早已不喜欢我 了的。”[7]显得如此的卑微、低贱。在《小团圆》中, 也
有“没有她们也会有别人，我不能与半个人类为敌”之类 的描写 [8]。在小团圆的背景上, 即使在盛九莉和邵之雍最 终分手的见面场合, 她和这个男人的过去“像长城一样, 在地平线绵延起伏”，尽管这长城已经没有用处了”[9]。两 人分开后, 张爱玲还寄了三十万元给他, 胡兰成也提到“都 是她寄钱来，现在最后一次她还如此。”[10]在这段婚姻关 系里, 张爱玲因精神的依附和对男性的崇拜, 她实在不能 算是完全意义上独立的女性。

张爱玲赴美后, 与赖雅结婚。两人结婚时, 丈夫年已 花甲，身体状况每况愈下，几近风烛残年。两人迁往旧金 山后, 赖雅曾明确地表示他对未来的收入并不能有多大的 贡献, 对此张爱玲表示赞同和理解。另外, 两人结婚才两 个月, 赖雅就因中风住进医院。其实他的中风史已有十余 年, 此前中风过两三次。同年, 他旧病又一次复发, 因面 部神经麻痹住院, 丈夫的疾病无疑给张爱玲的生活投下了 巨大的阴影。女人的肩上背负着养家、照顾丈夫等重任, 还要承受丈夫可能随时痽瘾在床或离她而去的风险。

在第二段感情关系中, 张爱玲充当的是家庭支柱的角 色。两性关系中“女强男弱”的格局决定了张爱玲必须要作 为完全独立的女性而生存。首先，她不顾两人年龄的巨大 差异及丈夫身体贏弱的现实状况, 勇敢地选择所爱的人, 并走入婚姻, 这就是拥有自我意识独立女性的理性选择。 其次, 她负担起挣钱养家的责任, 承担了经济来源的重担。 而且, 她还要以瘦弱的身躯照顾卧床的丈夫。这些现实的 窘境和困难让张爱玲无法依附于男性, 必须走出家门, 自 力更生。当然, 她的家庭地位也随之而升, 成为家里的顶 梁柱。在第二段婚姻中, 她是一个完整的人, 两人的婚姻 关系也从原来的倾慕肉体变为了精神的陪伴。婚姻生活的 不圆满让张爱玲从感情的依附蜕变到拥有女性意识、完全 独立的女性, 这是她人生真实的写照。在后期的小说创作 中, 张爱玲把自己不幸的婚姻投射到作品里, 书写了不少 与她境况类似、先进独立的女性形象。

\section{1. 金钱至上的女性}

“一顾倾人城，再顾倾人国。”[11]《倾城之恋》这部 小说看似一个完美无暇的传奇, 特别是配上作家张爱玲的 文笔。初看小说时, 仅看题目就会陶醉, 可逐渐展卷就会 发现那是一个荒诞不经的闹剧。

白流苏是上海一个典型的没落封建大家族女性。尽管 这样, 但是到了她这代, 不得不缩减日常开支。娶她是因 为其身份不同一般, 实际上她的容颜并不出众, 却有一种 差涩感。按照范柳原的话, 白流苏就是中国女人的典型, 她有着中国女性独特的魅力以及天生的務持。而小说的倾 城之恋, 并不在于她的美丽, 而是在于一座被战争摧毁了 的城市。小说深刻地揭示了女主人公物质至上的金钱观, 并予以了无情的嘲笑和辛辣的讽刺。正是这种与美学和浪 漫主题的矛盾元素, 奠定了小说伤感的悲剧基调。

作为大家闺秀的白流苏, 虽有涵养却无思想, 虽有城 府却无灵魂。她想靠男人过日子, 即使受尽凌辱, 也不愿 自食其力。所以, 尽管经过了战争的洗礼, 可白流苏仍未 因此而改变那种没落贵族女人特有的矝持神态。正是这 样, 作品塑造的人物形象显得真实而丰满。小说生动地描 
绘了那些不愿外出工作的人物, 那些人竭力维护自己在那 个时代所谓的高门大户的尊严和高贵。那“城市中的爱情” 的白流苏, 在一段失败的婚姻之后, 遭到了亲戚们的嘲笑。 在认识了浪漫的范柳原之后, 她开始对自己下注, 用自己 所理解的爱情来为自己博得一定的利益。范柳原和白流苏 都是自私的人, 他们之间的瓜葛都想从彼此身上得到实质 性的利益, 白流苏上等社会的情调吸引着范柳原, 而白流 苏则想要范柳原的钱财。白流苏精心谋划着, 但她自己清 楚地知道不能白白地让自己受损失; 范柳原根本不打算娶 白流苏为妻。他们彼此之间都看得清清楚楚, 后来当白流 苏确认经济已获得保障时, 就同意做范柳原的情妇, 这时 她一点掩饰都没有。在白流苏这样的人眼中, 玩弄他人是 可以获得幸福的。要不然, 他们都会成为待宰杀的羔羊, 没人愿意把自己的命运掌握在别人手里。这样一群大家国 秀们本该端庄聪慧美丽, 但是却不顾自身形象地把自己的 聪明才智用到夺取家产上。那些看似含蓄而和谐的人, 在 向别人展现自己婚姻的筹码, 在扭曲的生活中挣扎着, 却 终究无法逃脱被人生扭曲的悲惨命运。即使香港没有失 陷, 白流苏和范柳原也成不了夫妻。白流苏想要一个富裕 而有权势的丈夫, 财富和幸福与她是平等的。尽管她根本 没有爱的欲望, 但她渴望真爱, 这是不可避免的, 因为有 现实的需要。

由上可见, 张爱玲小说开展故事情节的主要线索是金 钱。要么显而易见, 要么隐而不显, 但人们会发现, 造成 女性的悲剧都是金钱导致的。作家小说生动地表达了那衰 败没落贵族女性所刻下的人性和心理。

除了金钱之外, 张爱玲塑造衰落家庭女性心理的另外 一种形式是爱无能, 是那种只有在某些极端情况下的心 理。而这种爱无能的冷漠, 在《花调》里淋漓尽致地体现 出来了。川娥的亲生母亲郑夫人在对待女儿爱情与婚姻的 问题上, 她的态度难以让人理解。当选择女婿时, 她说: “现在, 你不介绍她的男朋友, 她会介绍自己。她遇到了 一个好男人, 她自己带来的, 她不接受你的爱。”显然, 她并不是要选个能照顾女儿的女婿, 而像在谈一个不关已 的陌生人。她在乎的根本不是她女儿的幸福。女儿生病要 买药, 她想的是如果自己拿钱买药, 那就证明了她自己有 私房钱; 当女儿生命垂危时, 她想宁愿让女儿死去, 也不 愿把手放在丈夫手里。张爱玲用血腥的画笔绘制了旧世界 堕落贵族病态的真实画面。

\section{2. 沉默无助的女性}

作家笔下的许多女性非常复杂, 她们的人生充斥着无 奈。她们为名利而战, 仿佛这就是热爱生活, 但她们又哀 叹自己的灵魂已经牺牲了。小说中的女性生活在东西方文 明碰撞、社会动乱的时代。那个时代每个人的心中都存在 着“文明的终结感”和“对现实的悲观态度”。

作家小说中的爱情没有轰动一时, 但其爱情传奇却撕 裂了人性, 更令人震惊。作家说: “在这个时代, 旧事物 正在崩溃, 新事物正在增长。人们生活在一个时代, 但这 个时代正在像一个阴影一样下沉, 人们觉得他们已经被抛 弃了。”[12]
张爱玲从小就遭受父权的压榨, 不管父亲还是弟弟或 母亲, 她们对她的关心很少, 这使得她非常叛逆。她虽没 有被现实所压倒, 但最终她也没有逃脱当时妇女社会地位 低下的命运。这种矛盾心理在她的小说人物中有所反映: 小说人物都以一种被动的对立态度来面对命运留给她们 的痛苦。她们知道, 时代带来的变化是不可逆转的。不管 她们是否愿意, 她们唯一能做的就是在命运上做出妥协或 让步。

面对悲惨的命运, 她们要么假装这一切没有发生, 要 么任由自己四处游荡。《半生缘》中的顾谩桢嫁给了祝鸿 才, 她害自己失去了所有的人。期间, 不管是妹妹的受害, 情人的脆弱, 还是家人的不理解都把她自己推入了火坑, 最终她妥协了。秀珍非常善良, 温柔, 她身上具备作为新 女性的独立、知识和思想。她愿做工养活家人, 对待金钱, 她有着正确的价值观, 她有自己喜欢的人, 她自信开朗, 希望自己奋斗来度过自己的人生。顾谩桢的母亲顾太太是 一个被传统封建伦理禁锢的典型：一方面, 顾谩桢在母亲 身上看到了其意识行为的缺点, 并想要改变她; 另一方面, 她又自然而然地受到她母亲的影响, 而她自己却一点儿也 不知道。

谩桢的姐姐把她坑害了, 姐夫祝鸿才强奸了她。后来, 因为姐姐和母亲的胁迫, 她让步嫁给了祝鸿才。无论是软 弱, 还是内心存在着难以言明的痛苦, 那时她已经丧失了 自尊, 抛掉了自己的灵魂。后来, 谩桢离婚了, 可她还是 没有勇气去找自己真心爱的人。她在闹剧中摔倒了, 再也 没有站起来。虽然这些女性的形象是可憎的, 但某种程度 上她们又是可怜、可悲的。她们不仅埋葬自己的生命, 而 且为了满足他人的幸福还付出惨重的代价, 这种悲剧是那 食人社会特有的。

\section{3. 婚恋悲剧的女性}

从张爱玲的第一篇小说《沉香屑:第一炉香》到《五 四遗事》, 作家一直深切地关注着女性的悲剧命运。“特别 是善于在多种多样的 “性际关系” 中揭示女性存在的附庸 实质,及其广义上的 “娼女” 的真相。《第一护香》中的 梁太太及其侄女葛薇龙, 都在性际关系中产生了严重的倒 错:她们都经不住贵族男性(即使是没落的)的种种引诱, 都 将青春甚至整个生命,心甘情愿地舍予自己不爱或不值得 爱的男性”[13]

作品《沉香屑第一炉香》中的葛薇龙, 是一个女学生, 也是作者塑造的一个典型形象，她不仅善良美丽，还十分 纯洁天真。她家境落魄, 但很想上学, 在举家搬回上海时 无奈之下投靠了姑妈。她的姑妈做了一位百万富翁的妾, 家人反对也无济于事。当她的百万富商去世后, 留给了她 很多财产, 但她不得不用青春的游戏来填补心灵的空虚。 为了留住那些只看重她钱财的男人, 她想尽办法让年轻美 貌的葛薇龙成为她勾住那些男人的诱饵。初到姑妈家的葛 微龙对自己颇有自信, 可三个月后, 环境使她沉沦了, 也 迷上了姑妈这种生活。现实摧毁了她的梦想, 她逐渐放弃 了之前的尊严, 宁愿让自己不爱的男人得到自己。物质上 的巨大诱惑使得这个少女最后不仅失去了信仰, 甚至还丧 
失了人格。这一系列变化是对她试图幻想保持独立人格的 莫大讽刺。

尽管女性是独立而清醒的, 但她们终究无法摆脱父权 制的束缚。她们的自我认识并没有达到真正的醒悟, 也没 有获得真正的独立。

由此可见，作者对女性性欲、弱点的刻意表达，唯情 感是大, 而这就造成了她们的悲剧。在生活中, 生活与婚 姻的困蹇似乎都会在女性身上出现, 即便是职业上进的女 性, 也不感到自食其力的骄傲。而在生计操劳的困寒中, 男权时刻压迫着女性。在作家的小说中，几乎所有的女性 都在婚姻追随中生活, 在深层压迫下煎謷。

探讨生命的价值, 揭示人类生活的轨迹和终极目标, 可以说是作家把作品中的人物与她本人的精神相交了。作 者描绘的许多人物, 从出身低微的曹七巧、霓喜到名门出 生的白流苏、葛薇龙, 她们不顾一切的追随着属于自己的 人生阶梯。"葛薇龙"不惜一切代价牺牲父亲, 只是为了获 得姑姑的帮忙。白流苏毫不犹豫地下注, 逃离白公馆, 是 为了得到安定的婚姻。顾曼桢勤勤恳恳, 想要通过自身努 力以取得本该拥有的社会地位和经济条件。曹七巧费尽心 机, 极力满足自己青春的欲望, 想要获得物质财富上的享 受。但最后大都没有实现自己的人生目标, 走上了与自己 的愿望相反的路。葛薇龙最后不仅荒废了学业, 顾曼桢没 了事业, 霓喜不单没有钱, 还拖着一群没长大的孩子。曹 七巧得到的是黄金带给她的桎梏, 而白流苏虽然有了婚 姻, 其后果却是一个城市的覆灭。作家无疑是在告诉大家: 人生是凶恶无情的, 没有成功, 只有失败。那种妄想以奋 斗与努力来获得预想期望的女人简直是痴人做梦, 她越努 力就越违背她的目标, 就如一滩泥水深陷其中的人越使劲 挣扎就越下沉得深一样, 最突出明显的例子就是葛薇龙 了。《沉香屑第一炉香》一开头, 作者就特意写葛薇龙看 梁太太的房子，那花园如“一只金漆托盘”，树木“修剪得 齐齐整整”，看似整齐、干净、优雅的房子，葛薇龙想只 要她求学, 最终是可以实现这一切的。葛薇龙不是没有忧 虑, 可她凭着自己的意志和力量, 有信心 “走出泥潭而不 被玷污”。可是, 葛薇龙的一切奋斗都失败了, 一个纯洁 天真的女孩沦为妓女，背离了自己的初衷与期望。

人生的溃败不仅在于与自己的期望背道而驰, 还在于 平庸无常的人生意义以及对生存的厌倦。作家对时间、时 代意象和普通人度世状态的叙述也是对作品中人物精神、 心态的描述, 更是其对生存的感悟。作家时代的时间往往 是静止的。时间的相对静止体现了每个人生命的平庸枯 燥, 生活不是丰富多彩, 韶华容易死去, 就像“从水中钻 出来的歌又长又短; 它长, 又短......它还没有完成, 还没 结束呢。”[14]人们厌倦了单调重复的生活, 而这些甚至导 致人们“发疯”, 作家塑造的人物大都处在这个边界。例如, 内西一生中有四个不同种族的男人和五个不同种族的孩 子, 她不断重复着开始时混乱而被遗弃的生活; 梁太太等 一群人终日以酒买醉, 百般无聊, 靠追逐异性为生; 吴翠 远与崇祯萍水相逢, 谈情说爱, 虽然不会有结果, 但双方 都动了心, 投入了真情, 他们的生活没有颜色, 令人讨厌 而又无法挣脱。

作家笔下的青年女性通常显出平庸、无色调、缺乏趣 味。《红玫瑰与白玫瑰》中的孟烟丽大学毕业, 在十分封
闭的家庭中生活, 极度地缺少丈夫的爱, 她似乎与外界隔 绝了, 几乎没有与人诉说的机会, 空虚让她在一个裁缝身 上求得欢乐。“十八春”的顾曼桢纯真有进取心, 有着稳定 的事业和理想的那一半, 想靠自己奋斗去获得生存的价 值。在即将实现愿望的时候, 她却被亲友毁掉了, 失去了 男友, 丢掉了有前途的工作, 在万念俱灰中嫁给了自己讨 厌的祝鸿才, 由积极美好的向往陏入了深不见底的失望深 渊, 在生活的极度变化中, 使她感到单纯的积极的个人奋 斗竟变得毫无意义了。在险象环生中, 亲人间的挑拨作乱, 让这个正直向上的青年女性人生黯淡无光。作家认为, 人 的生活轨道发展规律是固定不变的, 生活被手操纵着, 人 们只能忍让: “生活是残忍的, 我们的欲望变得萎缩时, 悲伤涌上心头。”[15]在现实面前, 人的自信与向上精神微 不足道, 理想与追求也只是美好的幻想和无稽之谈。

落魄与平庸, 远不能浓缩作家理解生存的本质。如《金 锁记》里的姜长安在遭受了上洋学堂不成、婚恋失败的双 重打击后, 他的人生曙光开始暗淡。作家对人物的刻画没 有去描绘人物表情, 但姜长安的哀愁、无助、痛苦、绝望 却让读者有如身临其境。

\section{3. 女性精神特性产生的原因}

作品是“在传说中寻找普通人, 在普通人中寻找传 说”。在描写中, 作品勾勒出了平庸人在追求人生目标中 的挫折、失败、绝望, 也表现了一个普通人在平常生活中 的无望和凄惨, 反映了那个时代的社会悲剧。作品所反映 的悲剧其实是有路可循的, 作家发现二十世纪三、四十年 代国人精神信仰的彷得。

研究者特别关注作家张爱玲的家庭出身。家庭道德的 变化是作家思想形成的重要原因之一, 张爱玲的童年是不 幸的,她是在父母的争吵声中长大的。夫妻间长期的矛盾 和对立,使张爱玲的母亲痛下决心, 三十一岁时扔下两个年 幼的孩子,去英国留学学习西洋画,熟识了徐悲鸿、蒋碧薇 等名人, 离家留学, 实际上也是逃离不幸婚姻的藩篱。父母 失和家庭的孩子性格以内向型居多, “张爱玲也不例外,她 敏感多思,不愿与人交往,适应社会的能力较差, 文学、艺术 上有很高的天赋, 这是典型的内倾型性格, 在这样的家庭里 完全可以找到她性格形成的最终的原因。”[16]在作家那里, 正是她童年期的坎坷遭遇而真正决定了张爱玲的个性和 气质的,让她时时感受到世界“莽莽的威胁”,也让她后来的 作品充满了苍凉的悲剧氛围。[17]

当然更多的是社会时代变化因素的影响。二十世纪 三、四十年代, 中国社会是一个动荡不安的时代。正是在 这个混乱的时代，作家形成了对社会、生活、历史和现实 问题的基本看法。

动荡不安的社会, 人们漫无目的的生活, 没有颜色, 没有活力, 只有困苦, 就像《倾城之恋》中的那堵墙: “灰 砖砌成, 极高极高, 望不见边, 冷而粗䊅” [18]在张爱玲的 散文随处可见战争的阴霧,战争使张爱玲留学国外以期冲 出旧生活“围城”的理想破灭。浓重的战争阴影笼罩着人们, 个体显得那样的孤独、渺小和无奈。生命易逝, 朝不保 夕,“乱世的人,得过且过,没有真正的家”[19].摆在她面前的 
迫切人生课题, 是在乱世中如何保全性命, 寻得一个安稳的 人生避难场所。

在历史的成长中, 作家的家庭曾经有过一段时间的辉 煌, 只是在急速变革的大社会作家目睹自己显赫的贵族世 家经不起大变革的敲打, 逐渐衰败, 乃至没落。作为一个 成年人, 作家还记得儿时念诵的老清朝脸规上的泪水: “歌 女不知亡国之恨, 仍唱宫廷之花。”这犹如凛洌的寒风刺 痛着作家敏感而聪慧的心。贵族世家及其在朝代中鲜花锦 簇的光环早就没有了, 作家看到的不是随着改革变迁而枕 戈待旦、努力奋斗的人，而是及时行乐、步步走向沉沦的 一群人, 看到的是时代中没落及自私丑陃、可耻相残的人。 这是失去了价值观的一代, 从精神面貌到行为举止都存在 着阶级的沉溺。作家描写的那些角色对其他人来说是难望 其项背的，其中还表达了作家对自己家族长期养成的、根 深蒂固的不良习性的悲痛和唾弃。当把自己的人生追求转 化为创作激情并为史册记载文字的见证时, 作家慢慢地就 孕育了白公馆、姜公馆......而苍无悲凉就成了作家笔下流 淌着的主题思想, 生存的悲剧也就变成作家笔下人物的共 同命运。

\section{4. 结语}

综上所述, 作家以大写人生之椽笔塑造了中国二十世 纪三、四十年代一个十分鲜明的女性艺术群体, 作品对其 生存环境、人生态度、婚恋悲剧、独立意识等女性的精神 特性进行了入木三分的细致刻画, 其具体可感、栩栩如生 的艺术形象不仅青立于中国现代文学史册, 而且在世界文 学领域也产生了较大的影响。

创作如此宏大的艺术群体并非偶然, 这无疑与作家所 处的家庭背景、社会环境及其个人的文学天才密不可分。 张爱玲是一个奇特的女子, 聪慧过人。她曾经说: “我生 来就是写小说的”, “我对色彩、笔记和文字非常敏感”。当 作家树立了伟大理想时, 她便以细淢而通俗的小说文体打 开了中国现代文学的这扇大门。

出生于名门望族的张爱玲, 其人生悲剧是时代与现实 造致的。因为失落与绝望, 她把目标转向了历史, 希望把 痕迹留在历史中, 留下这阶级没落的见证, 把那创设的华 美比喻留下来, 把那灵动的人物留下来。凭借她那多彩的 作品, 张爱玲在中国现代文学史上耀眼夺目, 历史已经证 明了"张爱玲"的效应。在经历了地球震动的社会政治变革 之后, 国人再也没有闲情逸致了。作家张爱玲的创作开始 于平静, 没有更多的海浪, 结束也归于平静。

\section{参考文献}

[1] 间俊玲.简. 奥斯汀与张爱玲文学作品中女性人物比较 [J]. 语 文建设2015（35）。

[2] 张冬梅. 性格的扭曲精神的病态一一试从神经质性格视野 看张爱玲作品中的女性形象塑造[J].名作欣赏.2016(30)。

[3] 吴敬玲.情感的消解人性的异化一一张爱玲作品中女性形 象解读 $[\mathrm{J}]$.名作欣赏2017（05）。

[4] 高天瑞. 张爱玲小说中的女性意识探析 [J]. 长江丛刊 2019(29)。

[5] 胡兰成:今生今世[M]北京:中国长安出版社.2013。

[6] Yang Lili. Lack of Love and Tragedy of Zhang Ailing's Text [D].The University of Zheng Zhou,2007.

[7] 西岭雪.寻找张爱玲[M].长春: 时代文艺出版社.2014。

[8] 张爱玲.:张爱玲全集 $[\mathrm{M}]$. 北京: 十月文艺出版社,2012。

[9] 林幸谦.张爱玲“新作”《小团圆》的解读[J].中国现代文学研 究丛刊2009(04)。

[10] 胡兰成:今生今世[M].北京: 人民文学出版社.2002。

[11] 李延年. 北方有佳人 $[\mathrm{M}]$. 北京: 北京文艺出版社, 2003 。

[12] 张爱玲.自己的文章[M].北京: 京华出版社, 2005。

[13] 李继凯.论张爱玲小说中的女性异化[J].中国现代文学研究 丛刊1994（04）。

[14] 张爱玲.流言[M].北京:十月文艺出版社，2009。

[15] Yang Meifen. On the Tragic Implication of Zhang Ailing's Novels [D].The University of Xi An,2007.

[16] Yu Jing.A Study On Auspicious Omen of Tang ang Five Dynasties in Dunhuang Manuscripts[D].The University of Lan Zhou,2001.

[17] 张爱玲.关于《倾城之恋》的老实话 $[\mathrm{A}]$.张爱玲典藏全集(第 5 册) [M].哈尔滨:哈尔滨出版社,2003。

[18] 张华. 融合与疏离一论张爱玲的贵族气质与平民气质 $[\mathrm{J}]$. 吉 首大学学报(社科版)2007(04)。

[19] 张爱玲.张爱玲文萃·散文[M]. 北京: 文化艺术出版社, 2003。 